\section{Dialyse im Alter - wer darf, wer soll?}

\section{Einem besonders heikles Thema hat sich Prof. Ute Hoffmann vorgenommen, die Dialyse im Alter und die Frage: Wer darf, wer soll noch an die Dialyse ge- hen? Sie stellte zwei grundlegende Forderungen.}

Z unächst gilt es, die Prognose der älteren Patienten möglichst realistisch einzuschätzen. Hier eine Orientierungshilfe, zusammengestellt aus einschlägigen Veröffentlichungen der letzten zehn Jahre:

Das 1-Jahres-Überleben nach Dialysebeginn beträgt bei

_Patienten > 75 Jahren: $59 \%$

_Patienten $>80$ Jahren: $46 \%$,

_Patienten aus Pflegeheimen: 14-42\%.

_Ohne Dialyse bei 82-Jährigen immer-

hin noch $33 \%$.

Wie ist die zu erwartende verbleibende Lebensqualität? Diese hängt natürlich wesentlich von der individuellen Fitness und Lebenseinstellung ab. Es gibt hier die fidele Dame mit über 25 Diagnosen ebenso wie die bettlägrige Demente. Als Maß für die Einschätzung der individuellen Fitness wird meistens der FrailtyIndex nach Fried verwendet, als Maß für das Ausmaß der „Gebrechlichkeit“.

Für ältere Dialysepatienten ergaben sich in einer Studie laut Prof. Ute Hoffmann aus Regensburg folgende Vergleichswerte: Vor Einleitung der Dialyse betrug der Frailty-Fried-Index $48 \%$, nach einjähriger Dialysedauer bereits
$79 \%$. In diesem Index kommt zum Ausdruck, dass die Dialysepatienten über folgende Malaisen zu klagen begonnen haben: Gewichtsabnahme, verringerte Gehgeschwindigkeit, Müdigkeit, niedrige körperliche Aktivität oder Verminderung der Muskelkraft (Sarkopenie).

Dialysepatienten in einem Alter über 80 beklagen häufig kognitive Beeinträchtigungen, Depressionen und unkontrollierbare Schmerzzustände.

Und wie geht es den Patienten nach einem Jahr, bei denen keine Dialyse eingeleitet wurde? Eine Studie an 467 älteren Prädialysepatienten (Durchschnittsalter 82 Jahre) kam zu folgendem Ergebnis: Nach 12 Monaten wiesen $52 \%$ von ihnen stabile oder weniger Symptome auf, $58 \%$ berichteten über eine stabile oder bessere Lebensqualität. Werden die Patienten nach ihren persönlichen Lebenszielen befragt, was sie sich am meisten von der Behandlung erwarten, ist es die Autonomie - die Selbstversorgung ist ihr Kernbedürfnis.

Wann der Zeitpunkt für die Entscheidung gekommen ist, ob Dialyse oder konservative Therapie, liegt an den me-

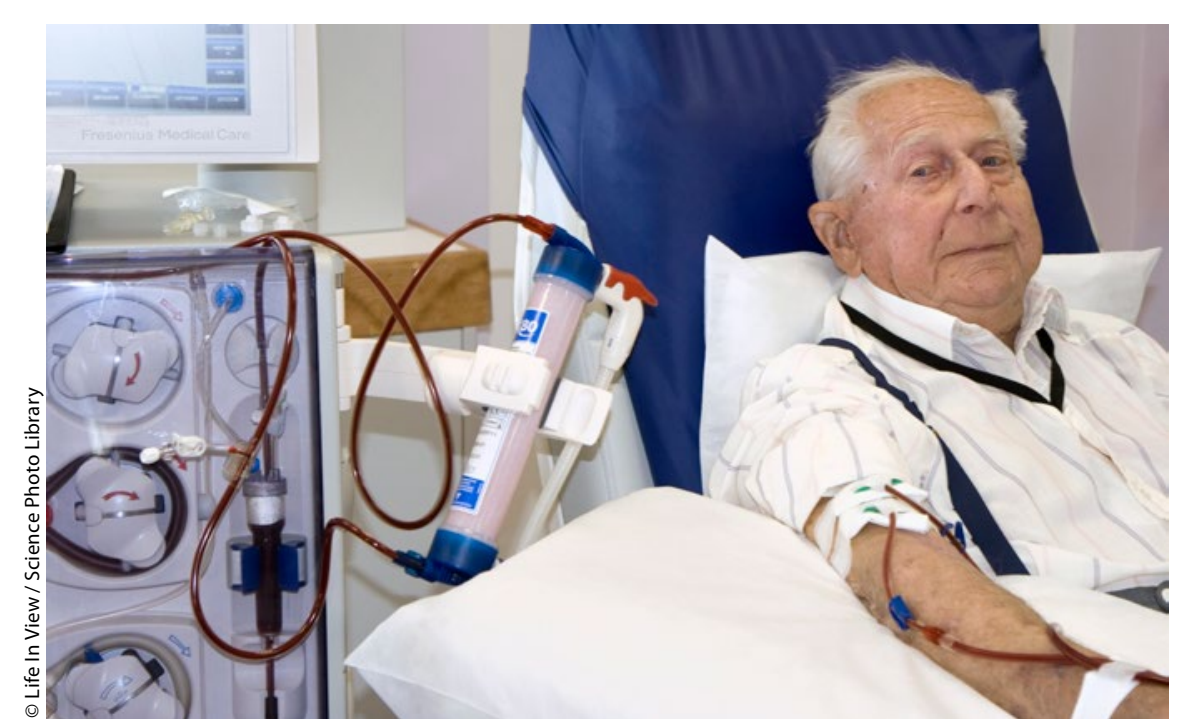

Klinische Parameter und der individuelle Zustand des Patienten entscheiden darüber, ob eine Dialyse-Therapie im Alter noch Sinn macht. dizinischen Parametern. Ausschlaggebend sind Urämiesymptomatik und Überwasserung (Ödeme, Pleuraergüsse).

Was dann vorgeschlagen wird, hängt auch vom Fitnessgrad und den Komorbiditäten ab. Den gebrechlichen, multimorbiden Patienten wird eher die Zentrumshämodialyse oder in weit fortgeschrittenem Stadium die assistierte Peritonialdialyse angeboten, für die noch "Fitteren“ kommt auch die Heimdialyse oder Heimperitonealdialyse in Betracht.

\section{Therapeutische Weichenstellung}

Der Entscheidungsprozess für die geeignete Therapieoption ist außerordentlich kritisch. Den Patienten müssen alle Optionen so dargelegt werden, dass sie auch verstanden werden und ihre Tragweite klar begriffen wird. Hoffmann kritisierte, dass es in Deutschland für diese lebensentscheidenden Gespräche keine strukturierten Aufklärungsbögen gibt. Sie hält sie für dringend erforderlich, aus ethischen Gründen wie auch zur Qualitätssicherung der Versorgung.

Noch ein zweiter, nicht minder leidenschaftlich vorgetragener Appell: Die Patienten sollten zu Beginn der Dialyse eine Patientenverfügung hinterlegen, und zwar alle. Bisher, so ihre Erfahrung, sind es höchstens $10 \%$ der Dialysepatienten, die eine solche verbindliche Verfügung vorweisen können.

An der Schwelle zur chronischen Dialyse kann mit einer Probedialyse den $\mathrm{Pa}$ tienten die endgültige Entscheidung erleichtert werden, etwa eine Dialyse auf drei Monate.

Die letzte Wegstrecke der Dialysepatienten kann Ärzte vor ethische Probleme stellen. Man sollte, wenn das Ende naht, nicht die Entscheidung treffen, die Dialyse „abzuschalten“ oder „abzusetzen“. Mit solchen Formulierungen werden vor allem die Angehörigen verstört und der Verdacht geschürt, man betreibe aktive Euthanasie. Hoffmann erklärt den Patienten wie den Angehörigen von Anfang an, dass es sich bei der Dialyse um eine aktiv lebensverlängernde Maßnahme handelt, die nicht jedes Mal von neuem durchgeführt werden muss. Wenn der Patient z. B. dann verlangt, so nicht länger leben zu wollen, kann die lebensverlängernde Maßnahme unterbleiben.

Dr. med. Jochen Aumiller 